Notfall Rettungsmed 2009 · 12:248-249

DOI 10.1007/s10049-008-1121-x

Online publiziert: 21. Mai 2009

๑) Springer Medizin Verlag 2009

H.R. Arntz $\cdot$ R. Somasundaram

Medizinische Klinik II, Kardiologie und Pulmonologie,

Charité Campus Benjamin Franklin, Berlin

\title{
Interdisziplinäre Notaufnahmen in Deutschland
}

\section{Die Entwicklung schreitet voran}

Während noch vor wenigen Jahren die Einrichtung interdisziplinärer Notaufnahmen an vielen Kliniken in Deutschland kein Thema war, wird ihr Potenzial nicht zuletzt wegen des zunehmenden ökonomischen Drucks, dem sich Krankenhäuser aller Versorgungsstufen stellen müssen, mehr und mehr erkannt. Abhängig von der Struktur einer Klinik werden etwa $30-50 \%$ aller stationärer Patienten über die Notaufnahmen aufgenommen, was ihre zentrale Bedeutung für die Prozesssteuerung einer Klinik unter qualitativen und ökonomischen Aspekten unterstreicht. Darüber hinaus ist dieser Bereich eine Art Visitenkarte. Eine gut funktionierende Notaufnahme ist in Anbetracht auch der Konkurrenzsituation von Krankenhäusern von nicht zu unterschätzender Bedeutung.

Im Gegensatz zu angloamerikanischen Kliniken beginnen sich in Deutschland allerdings erst jetzt Strukturen - oft als interdisziplinäre Not(fall)aufnahme bezeichnet - zu entwickeln. Diese zögerliche Entwicklung spiegelt sich auch in den uneinheitlichen Begrifflichkeiten wie

- Erste Hilfe,

- Rettungsstelle,

- Notfallambulanz

wider. Hier zeichnen sich aber die Termini zentrale oder interdisziplinäre Notaufnahme als die eingängigsten $a b$, wobei diese Diskussion noch nicht abgeschlossen ist.
Parallel zu dieser Entwicklung stehen erste Vorstellungen mit dem Ziel, das Berufsbild eines interdisziplinär tätigen Arztes für Notfallmedizin - ähnlich wie in vielen anderen europäischen Ländern - zu etablieren.

Das Leitthema dieses Heftes interdisziplinäre Notaufnahme will aktuelle Entwicklungen mit Beiträgen zu verschiedenen Aspekten auf diesem Gebiet begleiten.

Hogan et al. gehen mit ihrem Artikel kritisch sowohl auf die Möglichkeiten, aber auch auf die Grenzen zentraler Notaufnahmen ein. Letztere werden vor allem von den Fachgesellschaften gezogen. Wie „emergency physicians“ $z$. B. aus den USA erkennen lassen, wird diesen Ärzten im „emergency department“ vielfach nur eine äußerst eingeschränkte eigene Entscheidungskompetenz gestattet. Chest Pain Units, wie sie im Artikel von Post et al. beschrieben sind, könnten helfen, im Rahmen der zunehmenden Spezialisierung hier Abhilfe zu schaffen. Allerdings gelingt dies wiederum nur in inhaltlicher und örtlicher Zusammenarbeit mit einer interdisziplinären Notfaufnahme, wie das Mainzer Beispiel unterstreicht.

Der Erstversorgungsbereich mit chirurgisch/traumatologischem Schwerpunkt in einem Krankenhaus der Maximalversorgung stellt mit seinen speziellen Anforderungen an spezifische Diagnostik und gezielte chirurgische Erstmaßnahmen eine weitere Besonderheit dar. Zwei- fellos wird der Spezialisierungsgrad Notaufnahmebereich wesentlich von den Versorgungsmöglichkeiten der Spezialabteilungen abhängen und wird aber immer hohe Anforderungen an die dort tätigen Ärzte und Pflegekräfte stellen.

\section{( Der Notaufnahmebereich stellt hohe Anforderungen an die dort tätigen Ärzte und Pflegekräfte}

Die in aller Regel durch Pflegekräfte durchgeführte Erstsichtung und die damit verbundene Festlegung der Behandlungsdringlichkeit stellt einen entscheidenden Baustein im Prozess der Notfallversorgung dar. Allerdings ist dieser Prozess in Deutschland bisher wenig standardisiert (jede Klinik verwendet - wenn überhaupt - ihr eigenes System), aber mit der Etablierung international verwendeter und validierter klinischer Triage-Systeme zeichnet sich auch hier eine positive Entwicklung ab. Dabei stellt die Einbindung der Pflege in diesen Entscheidungsprozess, wie sie modellhaft in einem System wie der „Manchester triage" realisiert ist, ein schönes Beispiel dafür dar, wie gleichzeitig

- die Patientensicherheit erhöht,

- die Versorgungsqualität gesichert,

- die zentrale Rolle der Pflegekräfte in der Akutversorgung unterstrichen und

- die Mitarbeitermotivation gefördert werden kann. 
Wir stehen erst am Anfang der Entwicklung. Viele Fragen bleiben dementsprechend offen oder können in den Artikeln in diesem Heft von „Notfall + Rettungsmedizin" nur gestreift werden. Als Beispiel sei genannt, dass nicht definiert wird, welche Größe bzw. Struktur eine Klinik haben muss, um eine interdisziplinäre Notaufnahme einzurichten. Unbeantwortet bleibt zunächst die Frage, ob es zukünftig einen Spezialisten für Notfallmedizin geben wird und wer für diese Funktion am besten geeignet ist - nach unserer Überzeugung ein Arzt, der aufgrund seiner Ausbildung, Tätigkeit und Erfahrung ein besonders hohes Maß an Eigenkompetenz und dadurch Akzeptanz in den verschiedenen Fachdisziplinen mitbringt. Wir dürfen auf die Entwicklung der kommenden Jahre sehr gespannt sein und werden uns sicher bald wieder diesem wichtigen Themenkreis widmen.

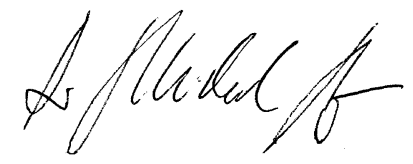

H.R. Arntz

\title{
Hier steht eine Anzeige.
}

\author{
Springer
}

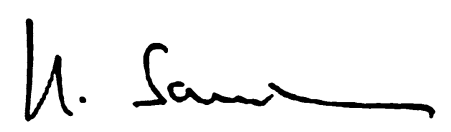

R. Somasundaram

\section{Korrespondenzadresse}

\section{Prof. Dr. H.R. Arntz}

Medizinische Klinik II, Kardiologie

und Pulmonologie, Charité

Campus Benjamin Franklin

Hindenburgdamm 30, 12200 Berlin

hans-richard.arntz@charite.de

\section{Literatur}

1. Hogan B, Brachmann M (2009) SWOT-Analyse einer Zentralen Notaufnahme mit Analyse der Erfolgspotentiale. Notfall Rettungsmed 4. DOI 10.1007/s10049-008-1123-8

2. Post F, Breuckmann F, Münzel T (2009) Die Rolle der Chest Pain Unit im Rahmen der Notfallaufnahme. Notfall Rettungsmed 4. DOI 10.1007/s10049008-1124-7 